\title{
La adopción y el uso de las tecnologías digitales en el profesorado universitario: un análisis desde la perspectiva del género y la edad.
}

\section{The adoption and use of digital technologies in university faculty: an analysis from the perspective of gender and age.}

\author{
Byron Geovanny Hidalgo-Cajo \\ Universidad Nacional de Chimborazo. Riobamba, Ecuador \\ bhidalgo@unach.edu.ec \\ Mercé Gisbert-Cervera \\ Universitat Rovira i Virgili. Tarragona, España \\ merce.gisbert@urv.cat
}

\begin{abstract}
Resumen
El éxito del proceso educativo mediado por la tecnología; depende, en gran medida, de la adopción y el uso de la tecnología digital (TD) por parte del profesorado. Teniendo en cuenta esta perspectiva en este artículo se presenta un diagnóstico multivariado sobre el nivel de adopción y uso de las TD, centrándose en las variables de género y edad. El estudio se llevó a cabo con una muestra de 280 docentes universitarios que respondieron el cuestionario denominado TAUT conformado por 46 ítems tipo escala de Likert distribuidos en cinco dimensiones: Propiedad y uso, Interés, Importancia, Percepción y actitud hacia las TD. La metodología aplicada en el estudio es cuantitativa basada en el método no experimental, descriptivo, correlacional, los datos fueron analizados a partir de la comparación de medias, utilizando pruebas no paramétricas. Los resultados revelan una autoevaluación positiva en la adopción y uso de las TD por parte del profesorado. Respecto a las variables analizadas, no existen diferencias significativas en función del género ni de la edad. Tampoco estas variables influyen a nivel de adopción y uso de las TD en el proceso enseñanza aprendizaje (E-A).
\end{abstract}

Palabras claves: Adopción y uso; Tecnologías Digitales; Educación Superior; género; edad.

\section{Abstract}

The success of the educational process mediated by technology depends, to a large extent, on the adoption and use of digital technology (TD) by teachers. With this perspective in mind, this article presents a multivariate diagnosis of the level of adoption and use of TD, focusing on the variables of gender and age. The study was carried out with a sample of 280 university teachers who responded to a questionnaire called TAUT made up of 46 Likert scale type items distributed in five dimensions: Ownership and use, Interest, Importance, Perception and attitude towards TD. The methodology applied in the study is quantitative based on the nonexperimental, descriptive, correlational method, the data were analyzed from the comparison of means, using non-parametric tests. The results reveal a positive selfassessment in the adoption and use of TD by the teaching staff. Regarding the variables analyzed, there were no significant differences according to gender or age. Neither do these variables influence the level of adoption and use of TD in the teaching-learning process (E-A).

Keywords: adoption and use; digital technologies; higher education; gender; age. 


\section{Introducción}

Las características de las TD y su integración a nivel global han originado cambios significativos, como la posibilidad de acumular información, su rápida transmisión, la desaparición de las barreras espaciales y temporales, el empleo de múltiples medios y soportes, son entre otras, algunas de las características que explican el gran potencial de cambio que aportan estas tecnologías (Cabezas et al., 2017).

La irrupción de las TD en las universidades, en las últimas décadas, está cambiando las formas de enseñar y aprender, ya sea por su simultaneidad, instantaneidad, interactividad, asincronía y ubicuidad (Flores et al., 2021), características que han abierto un nuevo abanico de oportunidades y demandas en la educación.

La adopción y uso de las TD pueden transformar los procesos educativos convencionales, y para ello las universidades han realizado importantes inversiones en equipamiento técnico, tecnológico y de conectividad, con el fin de que estas se integren en los procesos de enseñanza aprendizaje (E-A). A su vez y de manera paralela debe fomentarse en el profesorado su uso, así como el desarrollo de la competencia digital (Araiza y Pedraza, 2019), y que en consecuencia permitirá promover en el estudiantado el uso de las TD en sus procesos formativos para mejorar su éxito en el proceso educativo.

Por las razones que se acaba de mencionar es de suma importancia analizar la situación de la adopción y uso de las TD también en el contexto educativo superior y desde la perspectiva docente. En este sentido, existen estudios que revelan que la adopción y uso de las TD en los procesos educativos, todavía se enfrentan a una cierta resistencia por parte del profesorado (Kafyulilo et al., 2016 ; Teo, 2012). Las causas de esta resistencia son diversas, y varían entre la dificultad de acceso a los recursos, la falta de competencia técnica y pedagógica, la falta de materiales curriculares, y la falta de apoyo técnico y formativo (Barrantes et al., 2014).

Se puede mencionar que el éxito de la integración de las TD en el proceso (E-A) depende en gran medida del compromiso del profesorado, siendo necesario e importante contar con docentes competentes en el uso y manejo de la tecnología aplicada a la educación. No solamente para una eficaz transmisión de conocimientos, sino también con el objetivo de dotar al estudiante de herramientas que le permitan ser un individuo autónomo y social (Tarhini et al., 2014).

La integración de las TD en el ámbito educativo es una temática que tiene gran interés desde el punto de vista de la investigación. Por consiguiente, diversos estudios analizan su adopción y uso asociándolas a distintas variables tales como el género, la edad, los factores demográficos, la formación académica, la antigüedad (Luzardo et al., 2020), las características de los usuarios, la autoeficacia, o la satisfacción, y en el contexto institucional, las relacionadas con la disponibilidad de recursos y el ambiente de trabajo, entre otras. Estos estudios confirman y refutan la existencia de diferencias en la percepción y actitud que tiene el profesorado frente a la adopción y uso de las TD, según el contexto y la perceptiva del análisis (Ariza y Polanco, 2018; Lohbeck et al., 2018).

En particular el género ha resultado ser una variable influyente para medir el grado de aceptación que determina la intención de uso de las TD en la enseñanza (Pérez et al., 2016; Wong et al., 2012). Tal es así que investigaciones sobre las posibles diferencias de género en el uso y adopción de la tecnología en el contexto educativo, se han

La adopción y uso de las tecnologías digitales en el profesorado universitario: un análisis desde la perspectiva del género y la edad. Byron Hidalgo-Cajo y Mercé Gisbert-Cervera. Página 2 de 19 
incrementado en estas dos últimas décadas (Vázquez et al., 2017) con la aparición de internet y la configuración de la sociedad de la información y el Conocimiento (Liu y Sun, 2012; Wong et al., 2012).

Para Vera, Torres y Martínez (2014), la edad es un factor relevante en la adopción y uso de las TD, y aseveran que, a mayor edad del docente disminuye sus conocimientos en relación al uso de las tecnologías, estudio que es corroborado por Espinosa, Betancur y Aranzazu (2014) quienes encontraron diferencias significativas en cuanto al nivel de alfabetización informática, señalando que los docentes menores de 40 años tienen mejor nivel de conocimiento al emplear las TD en la docencia, contario a los mayores de 50 años. Como se ha expuesto diversos estudios evidencian diferentes resultados en relación al análisis de las variables género y edad, motivo por el cual se pretende conocer si estas variables influyen en la adopción y uso de las TD en el profesorado universitario desde otros entornos educativos.

\section{Metodología}

Para llevar a cabo el proceso de investigación, se empleó la metodología cuantitativa, con un diseño de investigación no experimental expostfacto-retrospectivo, transversal, descriptivo correlacional. El análisis de los datos se realizó por medio de la estadística descriptiva e inferencial (Hernández, 2014). Se estableció un nivel de significación p < 0.05 para todos los análisis.

\section{Muestra}

La población del estudio fue de 890 profesores, a quienes se aplico una muestra estratificada que corresponde a 238 profesores que representan a todas las facultades y carreras de la universidad, el nivel de confianza utilizado para determinar el tamaño de la muestra fue de $95 \%$, con un margen de error de $5 \%$ y una $\mathrm{p}=0.5$ y q $=0.5$, conjuntamente se aplicó un tipo de muestreo probabilístico, bajo la modalidad aleatoria y sistemática.

\subsection{Instrumento de recogida de datos}

La recogida de datos se llevó a cabo mediante el instrumento denominado Tipología Docente en la Adopción y Uso de la Tecnología digital TAUT (Hidalgo y Gisbert, 2021), y aplicado por medio de la técnica de la encuesta, en soporte electrónico, al profesorado universitario que conformó la muestra.

El instrumento aplicado fue de respuesta directa, pre-codificado y transversal. Está conformado por cinco dimensiones y 46 ítems implícitos, más un primer apartado de biodatos con 7 ítems, las dimensiones son: Propiedad y uso de la TD, se mide en función a la tecnología que posee el profesorado, incluyendo el propósito de uso, Interés de usar los recursos tecnológicos en el ámbito académico, se mide en función de la variable valoración de las TD en el proceso (E-A), Importancia al uso de las TD en el proceso (EA), se mide en función a la importancia de cada dispositivo tecnológico utilizado en el proceso educativo, Percepción de las TD como herramienta didáctica, se mide en función de la eficiencia y pertinencia de las TD en el proceso (E-A), y finalmente Actitud hacia

La adopción y uso de las tecnologías digitales en el profesorado universitario: un análisis desde la perspectiva del género y la edad. Byron Hidalgo-Cajo y Mercé Gisbert-Cervera. Página 3 de 19 
el uso de aulas virtuales (MOODLE) se mide en función de las variables utilidad y facilidad de uso.

Las dimensiones mencionadas, utilizaron la escala de valoración psicométrica tipo Likert, que permitió evaluar la opinión y actitudes del profesorado universitario, estas escalas fueron determinadas con una fuerza e intensidad lineal que se incrementa. De igual modo el cuestionario fue sometido a pruebas de fiabilidad mediante el Alfa de Cronbach, obteniendo un coeficiente de $(\alpha=0.813)$, que en efecto, según (McMillan y Schumacher, 2005), consideran una fiabilidad satisfactoria en coeficientes superiores a 0.787 .

A continuación, a modo de resumen se dan a conocer las cinco dimensiones más una de biodatos que forman parte del análisis, con sus diferentes ítems que las compone.

Biodatos. Datos de identificación: genero, edad, profesión, grado académico, años de experiencia docente, carrera, formación. (7 ítems).

Dimensión Uno. Propiedad (PU). Propiedad (PU_1) y uso de la TD (PU_2), se mide en función de la TD que posee o pretende adquirir el profesorado, incluyendo el propósito de uso de las TD. Se evalúan 10 ítems divididas en dos subdimensiones de 5 ítems.

Dimensión Dos. Interés (IU). Interés de uso de las TD, se mide en función a la valoración que brinda el profesorado a las TD en el ámbito pedagógico. Se evalúan 7 ítems.

Dimensión Tres. Importancia (MU). (MU_1) Importancia que brinda el profesorado a cada TD utilizada en el proceso (E-A) y la importancia que se brinda a las TD en el aula de clase (MU_2). Se evalúa en 10 ítems divididas en dos subdimensiones de 5 ítems.

Dimensión Cuatro. Percepción (EU). Percepción que tiene el profesorado de las TD como herramienta didáctica, medidas en función de la eficacia y la pertinencia de la TD en el proceso (E-A). Se evalúan en 7 ítems.

Dimensión Cinco. Actitud (AU). Actitud hacia el uso de las aulas virtuales, medida en función de la Utilidad (AU_1) y Facilidad de uso (AU_2). Se evalúa en 12 ítems, divididas en dos subdimensiones de 6 ítems.

\subsection{Análisis de datos}

El tratamiento estadístico de los datos se efectuó a través del programa Statistical Package for the Social Sciences (SPSS v.23), que permitió realizar el análisis mediante la utilización de estadísticos descriptivos e inferenciales. De manera descriptiva por medio del cálculo de estadísticos descriptivos básicos, relacionándolos con las características contextuales del profesorado como la edad y género, y de manera inferencial con el método de la prueba no paramétrica de U Mann-Whitney para la variable dicotómica género y la de Kruskal-Wallis para la variable edad. Todo ello con el fin de lograr describir los hechos que se plantean en las hipótesis propuestas y las posibles relaciones que existan entre las variables examinadas, en dependencia a la adopción y uso de las TD en el proceso (E-A) por parte del profesorado universitario.

El estudio en primera instancia realiza un análisis comparativo estratificado por edad y género del profesorado, seguido de un comparativo de las medias de cada una de las

La adopción y uso de las tecnologías digitales en el profesorado universitario: un análisis desde la perspectiva del género y la edad. Byron Hidalgo-Cajo y Mercé Gisbert-Cervera. Página 4 de 19 
dimensiones, en función al género. En la segunda fase se pretende dar respuesta a las diferencias que pueda existir en la adopción y uso de las TD por parte del profesorado en el proceso (E-A) en función de las variables modeladoras edad y género. Para cumplir con este fin se plantea las siguientes hipótesis $\mathrm{H} 1=$ Existe diferencias significativas entre hombres y mujeres con referencia a la adopción y uso de las TD por parte del profesorado universitarios y H0: No existe diferencias significativas entre hombres y mujeres en referencia a la adopción y uso de las TD por parte del profesorado universitario, de la misma manera en la variable edad se plantea las siguientes hipótesis $\mathrm{H} 1=$ Existe diferencias significativas en función de la edad en la adopción y uso de las TD por parte del profesorado universitarios y H0: No existe diferencias significativas en función de la edad en la adopción y uso de las TD por parte del profesorado universitarios.

\section{Resultados}

Este apartado de igual manera se estructura en dos subapartados. Por un lado, se presenta los resultados obtenidos en el análisis descriptivo de los datos, y por el otro, los obtenidos con el análisis inferencial.

\subsection{Análisis descriptivo}

Después de recolectados los datos del profesorado universitario se codificaron las respuestas de los reactivos y se aplicaron cálculos estadísticos.

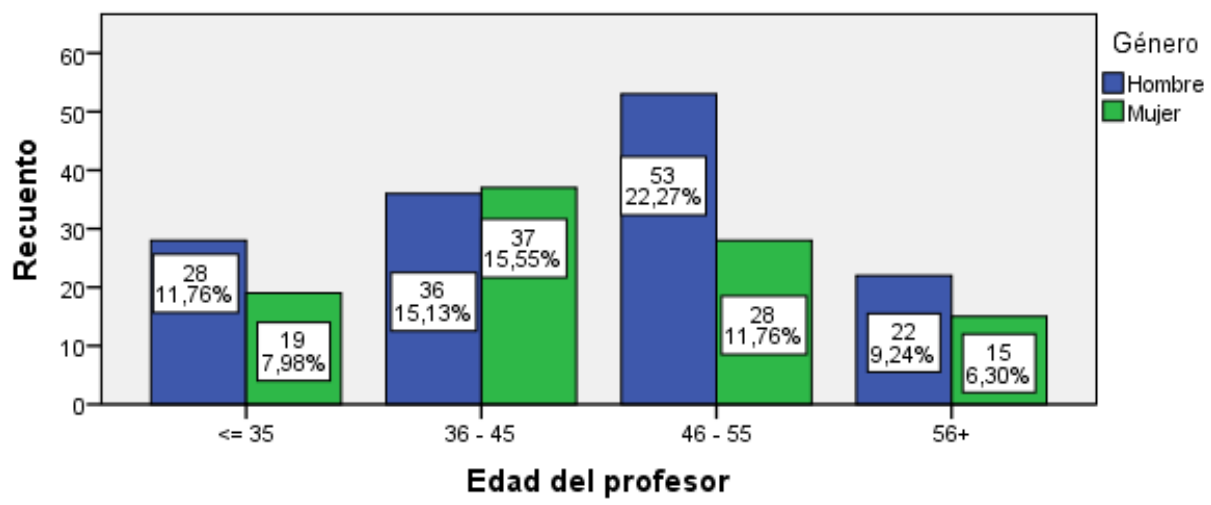

Gráfico 1. Análisis comparativo estratificado por edad y género

En este apartado se evidencia que 238 docentes involucrados en la investigación, 139 $(58,4 \%)$ son hombres y $99(41,6 \%)$ mujeres, con un promedio de edad de 44 años, las edades de (36 - 45) y de (46 - 55) años, son los rangos donde se concentran la mayor cantidad de docentes tanto hombres como mujeres, estos resultados permiten tener una visión prospectiva relacionada con el estudio de Matas (2016) quien afirman que estas edades demuestran el grado más intenso de penetración de las tecnologías.

De igual modo mediante el análisis descriptivo de todas las dimensiones de cuestionario TAUT sobre la adopción y uso de la TD en el proceso (E-A) por parte del profesorado universitario, se puede mencionar que la autoevaluación se realiza en un rango del 1 a 5

La adopción y uso de las tecnologías digitales en el profesorado universitario: un análisis desde la perspectiva del género y la edad. Byron Hidalgo-Cajo y Mercé Gisbert-Cervera. Página 5 de 19 
( 1 bajo a 5 alto), mediados por el género del profesorado. Los resultados se pueden apreciar en el gráfico 2.

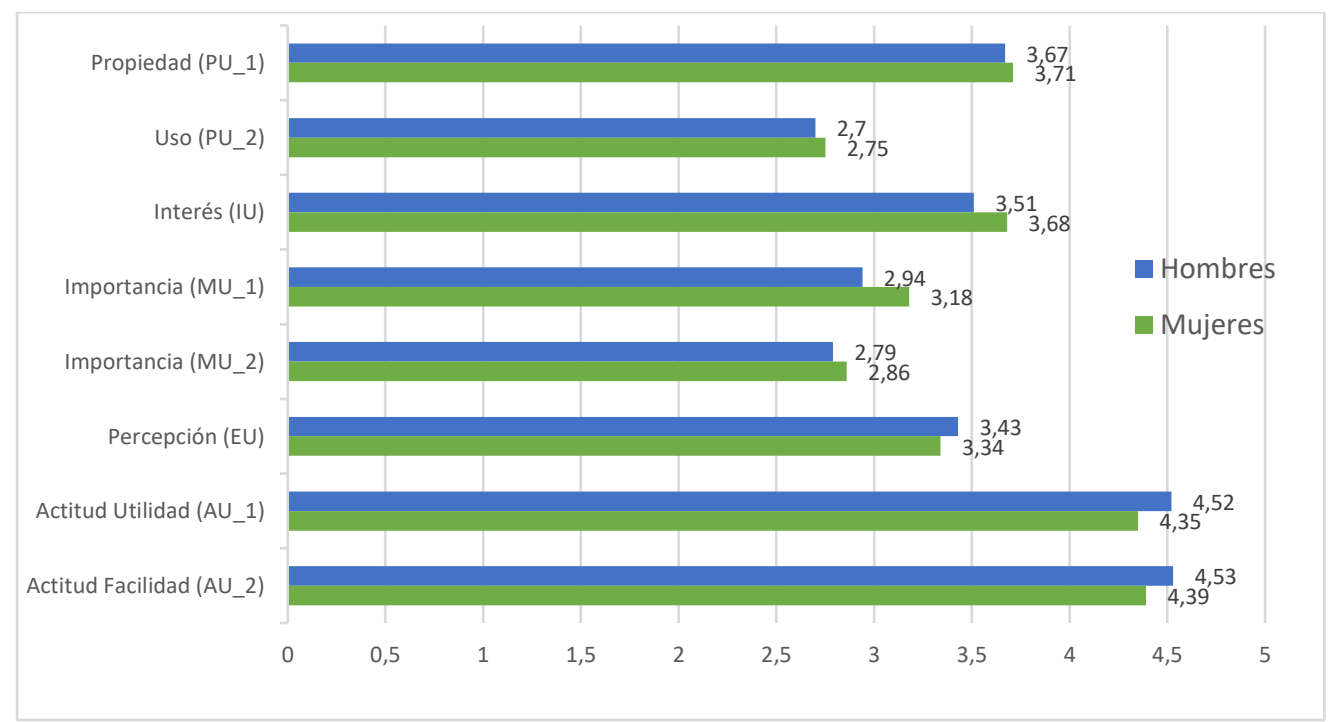

Gráfico 2. Estadísticos descriptivos de las dimensiones TAUT

Una vez realizado el análisis descriptivo de manera general, se obtiene la media de cada una de las dimensiones, observándose una alta aceptación de las TD por parte del profesorado, tanto en hombres como mujeres. Este resultado manifiesta, respecto a la actitud que muestra el profesorado en adoptar, usar e integrar las TD en el proceso (E-A), esta actitud y autoeficacia percibida por las tecnologías, son variables que pueden influir en su adopción y uso en la práctica educativa, de tal manera que el profesorado gradualmente lograra adquirir competencias digitales (INTEF, 2017). Por otra parte, se enfatiza una relación de valoraciones entre hombres y mujeres, sin embargo, la información disponible no permite confirmar o refutar la hipótesis planteada para lo cual se procede analizar individualmente cada uno de los ítems que contempla las dimensiones y subdimensiones del TAUT mediante un análisis inferencial.

\subsection{Análisis inferencial}

A continuación, se exponen los resultados comparando las medias en función de las variables moderadoras género y edad, dentro de este marco se presenta la existencia o no de diferencias significativas encontradas a partir del análisis estadístico realizado.

\section{- Variable moderadora Género}

Para dar respuesta a la primera Dimensión, "Propiedad y uso de las TD", que se refiere a ¿conocer si existe diferencias significativas en función del género en relación a la propiedad y uso de las TD?, se procede a analizar los diferentes ítems que contempla esta

La adopción y uso de las tecnologías digitales en el profesorado universitario: un análisis desde la perspectiva del género y la edad. Byron Hidalgo-Cajo y Mercé Gisbert-Cervera. Página 6 de 19 
dimensión, las mismas que son valoradas individualmente y están basadas en el cuestionario TAUT encontrando los siguientes resultados en el gráfico 3.

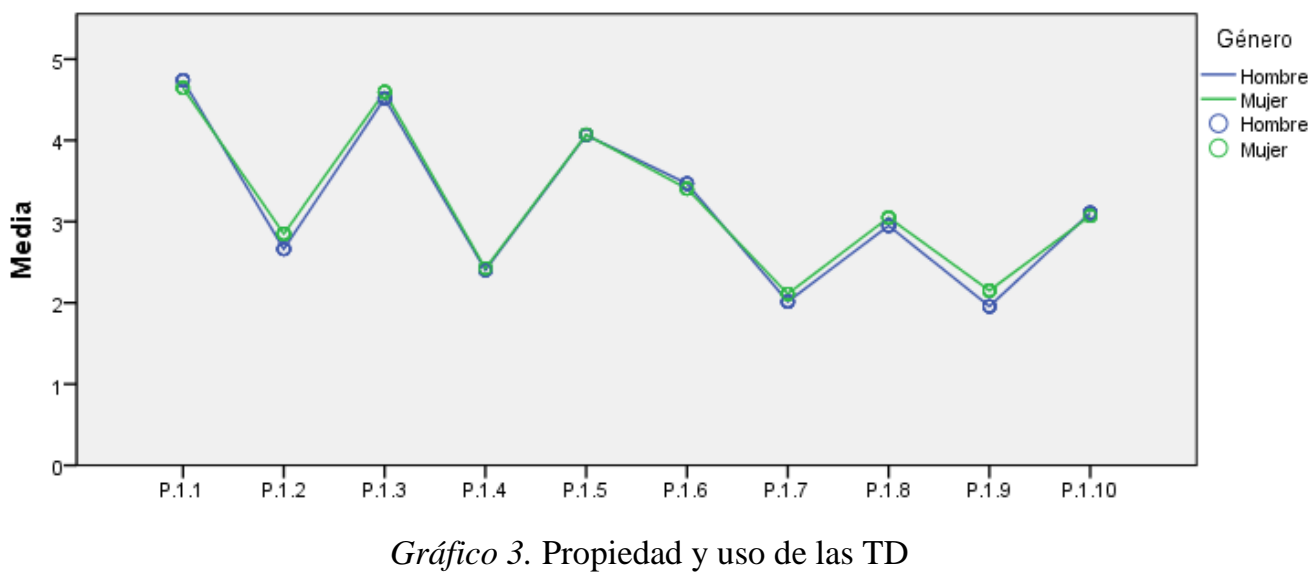

En el gráfico 3, se pueden apreciar los resultados de las valoraciones en referencia a la media a partir de la variable género, siguiendo con el análisis se aplica la prueba de Levene para obtener el p-valor de todos los ítems de esta dimensión, los cuales fueron superiores a $\alpha=0.05$ permitiendo inferir que las varianzas son iguales, del mismo modo los valores de significancia calculados superaron a $\alpha=0.05$, en consecuencia se acepta la hipótesis nula, y se concluye que no existe diferencias significativas entre hombres y mujeres en lo concerniente a la propiedad o la pretensión de adquirir las TD a corto plazo, como en el propósito de uso que el profesorado brinda a las TD.

Con respecto a la Dimensión dos. "Interés en las TD para el uso académico", se puede apreciar que las varianzas en todos los ítems de esta dimensión son iguales. Así como también se puede observar que no existen diferencias significativas entre hombres y mujeres en los siguientes ítems; valorar el uso de la biblioteca institucional (P.2.1; sig. bilateral=0.237), así como también las herramientas web para bibliografía (P.2.3; sig. bilateral=0.817), los medios de comunicación mediados por las TD (P.2.4; sig. bilateral $=0.874)$, los libros y textos digitales $($ P.2.5; sig. bilateral $=0.335)$ y el contenido de las asignaturas que están disponibles externamente de la institución (P.2.6; sig. bilateral $=0.149$ ), por el contrario la valoración en el uso de las aulas virtuales (P.2.2; sig. bilateral=0.017), así como las simulaciones o juegos educativos (P.2.7,sig bilateral=0.38), presentan diferencias significativas entre hombres y mujeres, como se puede apreciar en el gráfico 4.

La adopción y uso de las tecnologías digitales en el profesorado universitario: un análisis desde la perspectiva del género y la edad. Byron Hidalgo-Cajo y Mercé Gisbert-Cervera. Página 7 de 19 


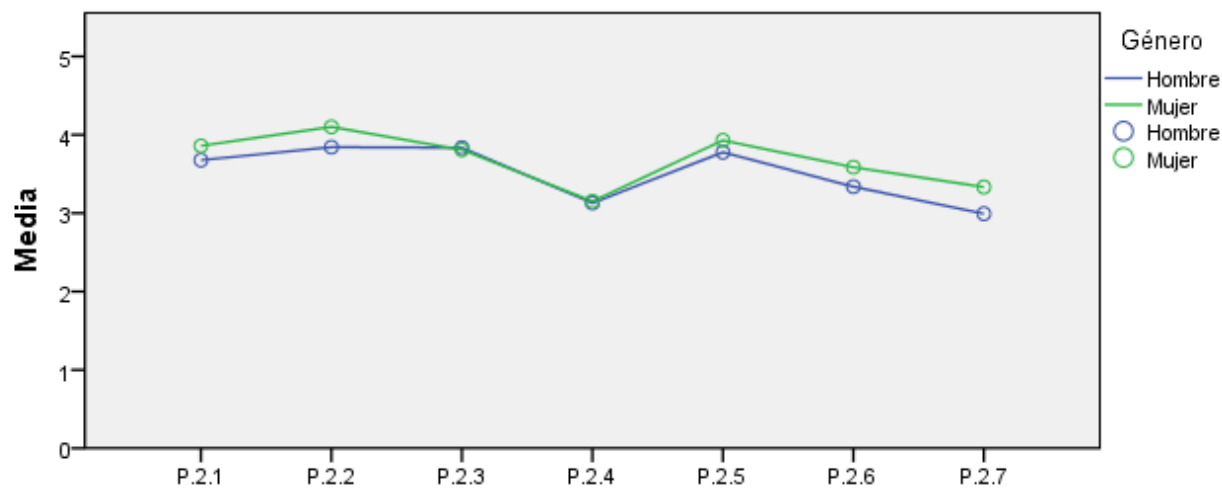

Gráfico 4. Interés en las TD para el uso académico

Siguiendo con el análisis, en la dimensión tres. "Importancia que brinda el profesorado a cada TD utilizada en el proceso (E-A)", se puede indicar que las varianzas en todos los ítems de esta subdimensión son iguales, así como también no existe diferencias significativas entre hombres y mujeres en los siguientes ítems; la computadora portátil o notebook (P.3.1; sig. bilateral=0.281), la Tablet o Ipad (P.3.2; Sig. bilateral=0.510) y el computador de escritorio o PC (P.3.5, sig. bilateral=0.728), por lo contrario se puede evidenciar la existencia de diferencias significativas en el smartphone (P.3.3; Sig. bilateral $=0.034)$, y el lector de libros digitales (P.3.4; sig. bilateral=0.015).

Por otro lado, se analiza la subdimensión, "Importancia que brinda el profesorado a las TD en el aula de clase", los resultados evidencian la igualdad de varianza en todos los ítems, y se determina que no existe diferencias significativas entre hombres y mujeres, sin embargo, se puede mencionar que sus valores de media obtenidos son los más bajos de todas las dimensiones, como se puede visualizar en el grafico 5.

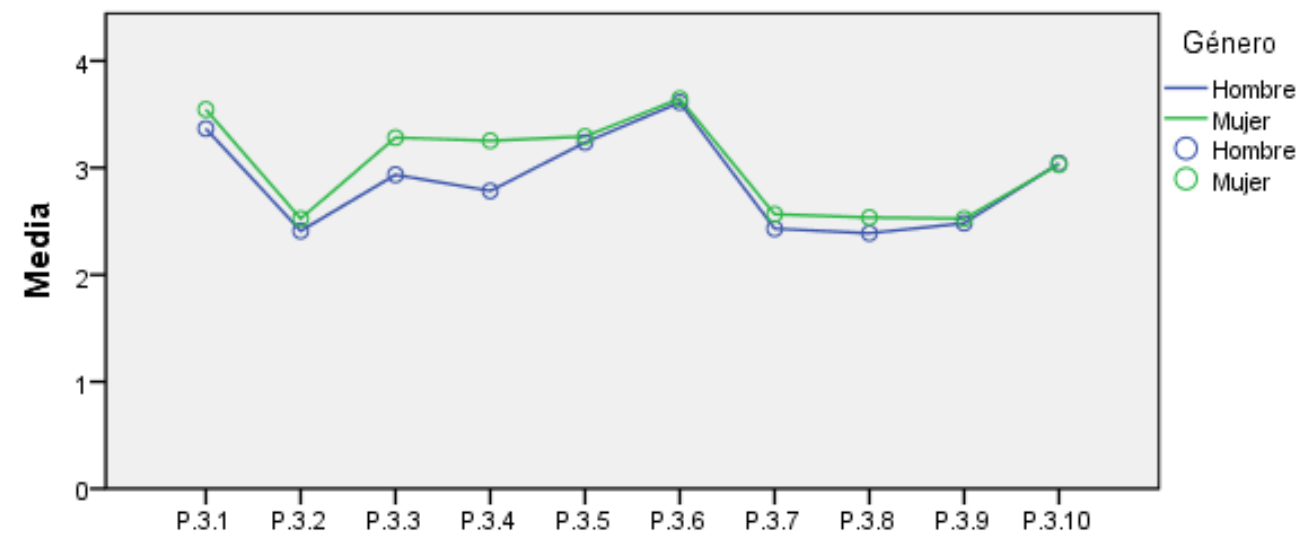

Gráfico 5. Valoración de las TD en el proceso educativo

Dimensión cuatro. "Percepción que tiene el profesorado de las TD como herramientas didácticas". Esta dimensión se mide en función de la eficacia y la pertinencia de la TD en el proceso (E-A). El análisis arroja los siguientes resultados; las varianzas en todos los ítems son iguales. No existen diferencias significativas entre hombres y mujeres, es así que la autoevaluación se manifiesta positivamente respecto a la percepción del

La adopción y uso de las tecnologías digitales en el profesorado universitario: un análisis desde la perspectiva del género y la edad. Byron Hidalgo-Cajo y Mercé Gisbert-Cervera. Página 8 de 19 
profesorado en cuanto a alcanzar de mejor manera los objetivos educativos, consiguiendo realizar búsquedas de información en internet que se adapten a las necesidades educativas, creando recursos didácticos, con una mayor selección de contenidos que se adecúan al desarrollo de la asignatura, a su vez la comunicación e interacción por medio de las TD aportan al proceso educativo, siendo la institución la que fomenta el uso de las TD como herramienta didáctica en el quehacer educativo, como se puede apreciar en el grafico 6.

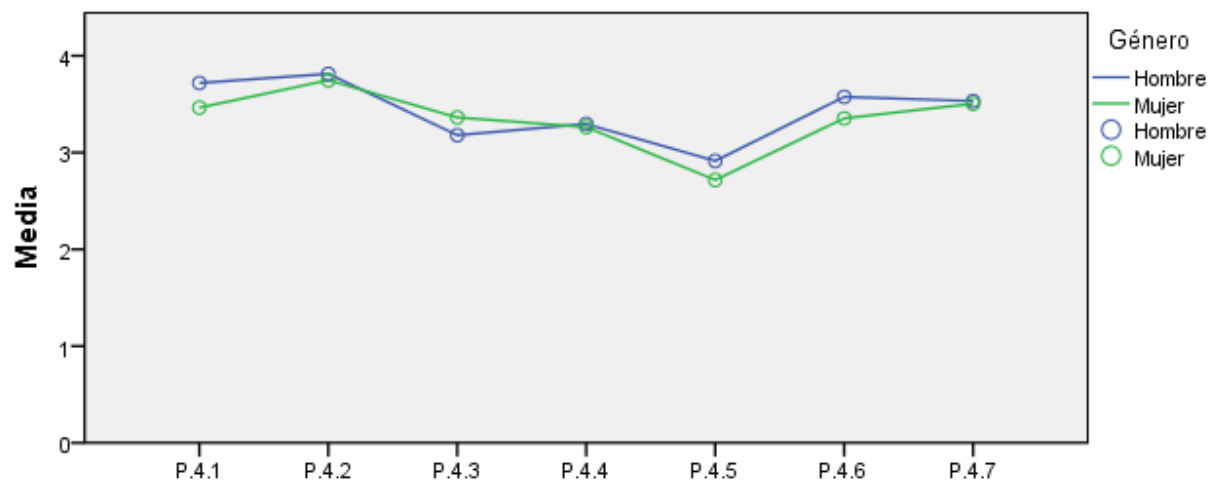

Gráfico 6. Ventaja que brinda las TD como herramienta didáctica

Dimensión cinco. "La Actitud hacia el uso de las aulas virtuales". Esta dimensión es medida en función de la utilidad y facilidad de uso percibido de las aulas virtuales. Los resultados reflejan una valoración alta tanto en las mujeres como en los hombres, como se presenta en el grafico 7 .

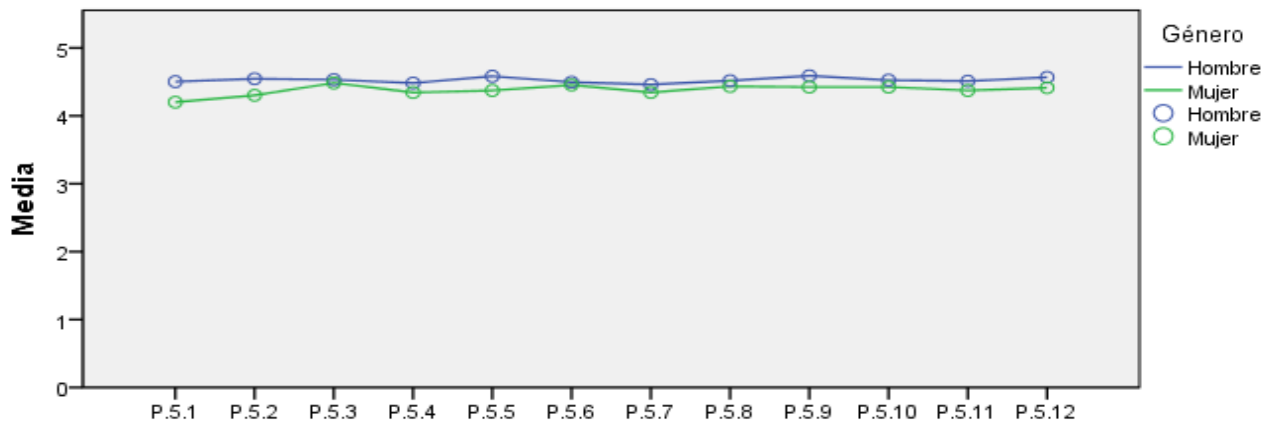

Gráfico 7. Utilidad y Facilidad de uso de las aulas virtuales (MOODLE)

Haciendo referencia a la subdimensión "Utilidad percibida por el uso de las AV", sus varianzas son iguales, se determina la existencia de diferencias significativas entre hombres y mujeres en los siguientes ítems; las AV ayudarían a tener mayor interacción con los estudiantes (P.5.1; sig. bilateral=0.014), mejorando el desempeño académico (P.5.2; sig. bilateral=0.029), lo cual facilitaría la realización del trabajo académico (P.5.5; sig. bilateral $=0.029$ ) . Por lo contrario, no existe diferencias significativas en función del género en; Usar las AV podría incrementar mi productividad (P.5.3; sig. bilateral=0.629), la efectividad (P.5.4, Sig. bilateral=0.165), y utilidad en el trabajo académico (P.5.6; sig. bilateral $=0.672)$.

La adopción y uso de las tecnologías digitales en el profesorado universitario: un análisis desde la perspectiva del género y la edad. Byron Hidalgo-Cajo y Mercé Gisbert-Cervera. Página 9 de 19 
En la subdimensión "facilidad de uso de las AV", sus varianzas son iguales, no existe diferencias significativas en todos los ítems, ya que el profesorado percibe que aprender a utilizar las AV seria fácil (P.5.7; Sig. bilateral=0.300), así como su acceso para hacer lo que hay que hacer (P.5.8; sig. bilateral=0.391), la interacción con las AV seria clara y entendible (P.5.9; sig. bilateral=0.073), percibiendo al AV como un sitio flexible para interactuar (P.5.10; sig. bilateral=0.281), lo cual le llevaría al profesorado a ser un experto en el uso de las AV (P.5.11; sig. bilateral=0.201), ya que lo encuentra fácil de utilizar (P.5.12; sig. bilateral=0.116).

\section{- Variable moderadora Edad}

El objetivo pretendido en esta sección fue determinar si existe una asociación significativa entre la edad del profesorado y la adopción, uso e integración de las TD en el proceso (EA), de tal manera se procede a realizar el análisis estadístico inferencial mediante la prueba de Kruskal Wallis, para lo cual se plantea las siguientes hipótesis.

H1 = Existe diferencias significativas en función de la edad en la adopción y uso de las TD por parte del profesorado universitario y H0: No existe diferencias significativas en función de la edad en la adopción y uso de las TD por parte del profesorado universitario.

Para poder dar luz a estos cuestionamientos se procede a analizar la primera dimensión, Propiedad y uso de la TD relacionada con ¿conocer si existe diferencias significativas en función de la edad con respecto a la Propiedad y uso de la TD por parte del profesorado?, los diferentes ítems, valoradas individualmente, que contempla esta dimensión, se aprecian en el gráfico 8.

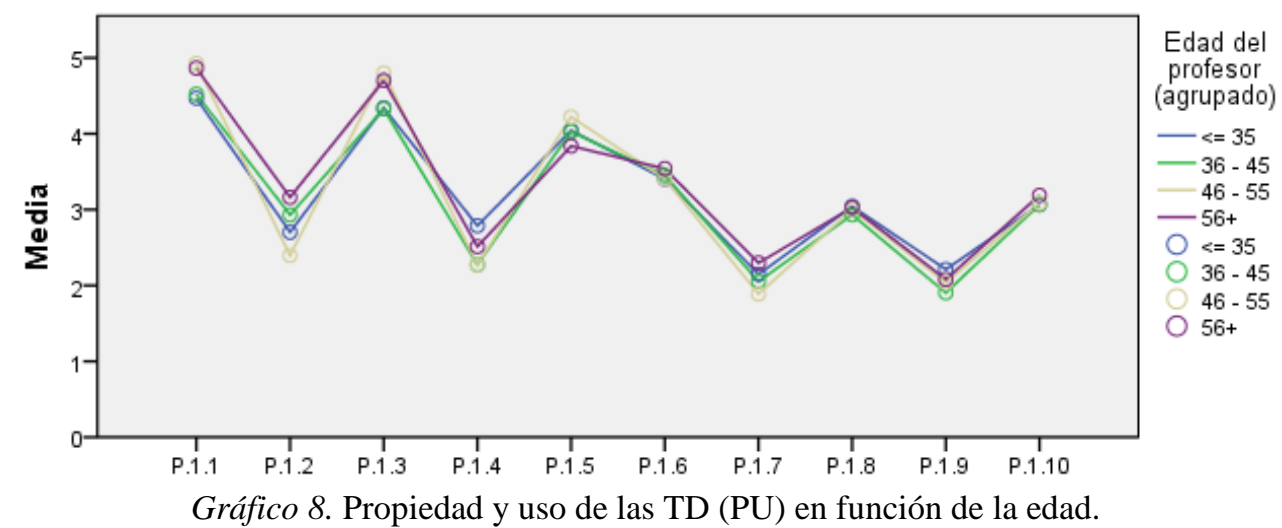

Con respecto a la "Propiedad o pretensión de adquirir la TD", se puede apreciar que la edad comprendida entre (46 - 55 años), y más de 56 años son los dos intervalos en los que se sitúan los profesores que mayoritariamente poseen o pretender adquirir las TD, sin embargo los intervalos de edad comprendidos entre (36-45 años) y los menores de 35 años, también tiene la misma actitud pero en una menor escala, esta información revela que existe una elevada actitud en poseer o pretender adquirir a corto plazo las TD en todos los intervalos de edad del profesorado. Sin embargo, el análisis estadístico demuestra que existe diferencias significativas en poseer o pretender adquirir a corto plazo las TD en los siguientes ítems; en el computador portátil (P.1.1; Sig. asintótica=0,015), y el Smartphone (P.1.3; Sig. asintótica=0,045), por lo contrario; no existe diferencias significativas en

La adopción y uso de las tecnologías digitales en el profesorado universitario: un análisis desde la perspectiva del género y la edad. Byron Hidalgo-Cajo y Mercé Gisbert-Cervera. Página 10 de 19 
función de la edad; en la Tablet (P.1.2; Sig. asintótica=0,113), el Lector de libros digitales (P.1.4; Sig. asintótica=0,254) y la PC (P.1.5; Sig. asintótica=0,644).

En cuanto a la subdimensión "Propósito de uso que el profesorado brinda a las TD", el análisis de los datos manifiesta que todos los intervalos de edad en los que se agrupa al profesorado universitario se mantienen en un equitativo propósito de uso de las TD en el proceso (E-A), en consecuencia, la sig. asintótica calculada en cada una de las TD evaluadas supera el 0.05 por lo que se puede concluir que no existe diferencias significativas en función de la edad en esta subdimensión.

Al analizar la dimensión dos. "Interés de uso de las TD", se evalúa en función de la valoración que asigna el profesorado a las TD en el proceso (E-A). En este sentido el profesorado ( $<=35$ años) y mayores de 56 años de edad son los que valoran positivamente el uso de las TD en el proceso (E-A). Del mismo modo los intervalos de edad comprendidos entre los (36-45) y (46-55 años) son los que tienden a valorar en menor escala esta dimensión, ver gráfico 9. El estudio determina que si existe diferencias significativas en función de la edad del profesorado y el interés de uso de las TD en el proceso (E-A), en los siguientes ítems; valorar el uso de la biblioteca virtual institucional (P.2.1; Sig. asintótica=0,016), libros y textos digitales (P.2.5; Sig. asintótica=0,009), herramientas web para bibliografía (P.2.3; Sig. asintótica=0,041) y simulaciones o juegos interactivos (P.2.7; Sig. asintótica=0,016); por lo contario no existe diferencias significativas; en usar las aulas virtuales (P.2.2; Sig. asintótica=0,143), como tampoco en los medios de comunicación mediados por las TD (P.2.4; Sig. asintótica=0,387), y el contenido de las asignaturas que están disponibles externamente de la institución (P.2.6; Sig. asintótica=0,137).

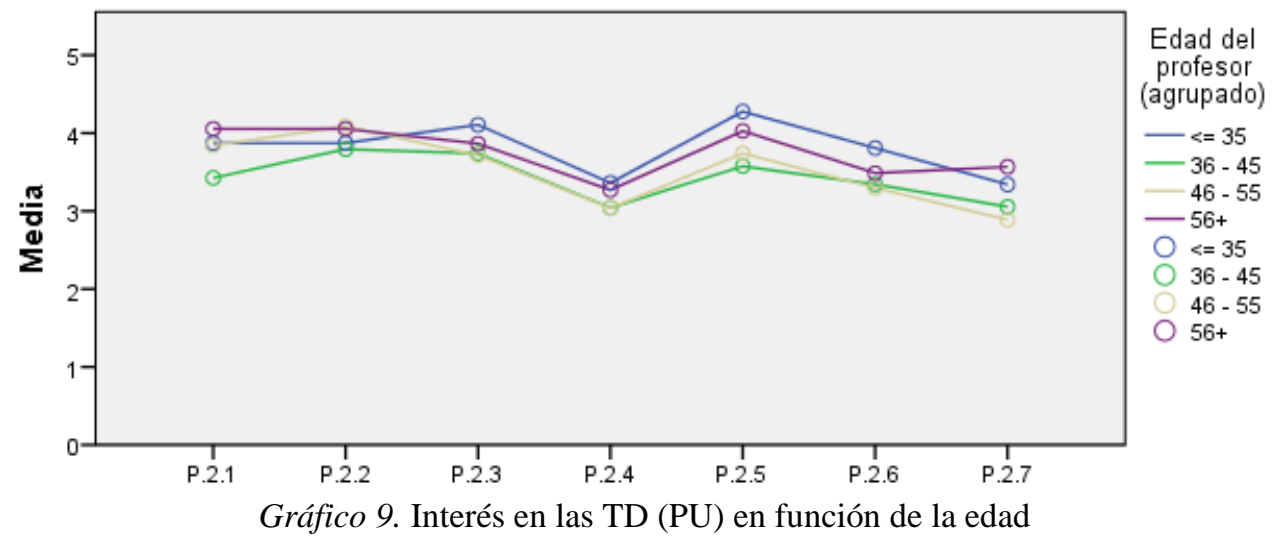

En la Dimensión tres se analiza, la "Importancia que brinda el profesorado a cada TD utilizada en el proceso (E-A)". El análisis determina que el profesorado mayor a los 56 años confiere mayor importancia al uso de las TD en el proceso (E-A) seguidos de los menores o iguales a 35 años, sin embargo, los intervalos de edad comprendidos entre los (46-55 años) y (36-45 años) tienden a dar menor importancia al uso de las TD. El estudio manifiesta que si existe diferencias significativas en función de la edad y la importancia que brinda el profesorado a la Tablet (P.3.2; Sig. asintótica=0,026), los libros digitales (P.3.4; Sig. asintótica=0,009) y el computador de escritorio (P.3.5; Sig. asintótica=0,013), por lo contrario no existe diferencias significativas en la computadora portátil (P.3.1; Sig.

La adopción y uso de las tecnologías digitales en el profesorado universitario: un análisis desde la perspectiva del género y la edad. Byron Hidalgo-Cajo y Mercé Gisbert-Cervera. Página 11 de 19 
asintótica=0,196) y el smartphone (P.3.3; Sig. asintótica=0,162), tal como se demuestra en el Gráfico 10.

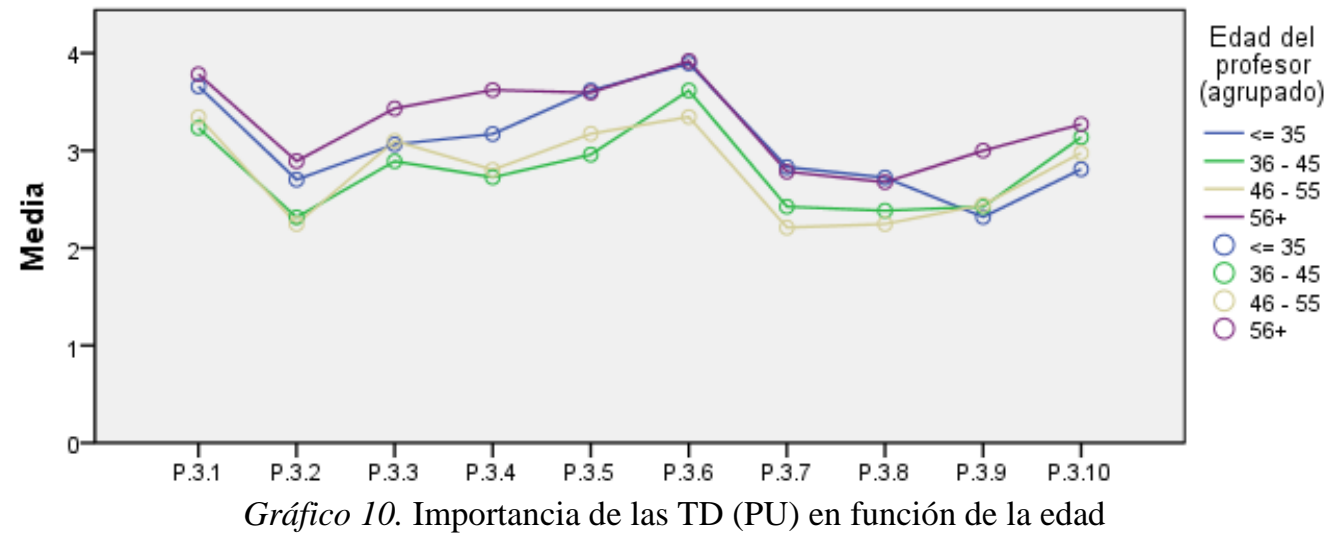

En referencia a la subdimensión, "Importancia que el profesorado le brinda al uso de las TD en el aula de clase", el análisis muestra que el profesorado mayor a 56 años, seguido por los menores o iguales a 35 años le dan importancia al uso de las TD en el aula de clase. No obstante, los intervalos de edad comprendidos entre (46-55 años) y (36-45 años) tienden a dar menor importancia al uso de las TD en el aula de clase. De manera similar se analiza individualmente cada TD en función de la edad y los resultados reflejan, diferencias significativas en cuanto a la importancia que se le da al uso del computador portátil en el aula de clase (P.3.6; Sig. asintótica=0,031) así como también de la Tablet (P.3.7; Sig. asintótica=0,008), por el contrario no existe diferencias significativas respecto al uso del Smartphone (P.3.8; Sig. asintótica=0,120), del lector de libros digitales (P.3.9; Sig. asintótica $=0,108)$ y el computador de escritorio (P.3.10; Sig. asintótica=0,401) en el aula de clase.

En cuanto a la Dimensión cuatro. "Percepción que tiene el profesorado de las TD como herramienta didáctica". Los resultados mencionan que el profesorado $(<=35)$, seguido por los del intervalo (36-45 años) son que valoran positivamente la eficacia y la pertinencia de las TD como herramienta didáctica, al contrario de los intervalos de edades de (46-55) y mayores a 56 años. Con el análisis inferencial se puede observar que existe diferencias significativas mediadas por la edad en lo referente a los ítems; información que el profesorado encuentra en Internet (P.4.2; Sig. asintótica=0,000), como también a los recursos didácticos y educativos que proporciona las TD (P.4.5; Sig. asintótica=0,001). Por lo contrario no existen diferencias significativas en los ítems; la TD le permite conseguir mejorar los objetivos educativos con los estudiantes (P.4.1; Sig. asintótica $=0,622$ ), así como también, las TD permiten la creación de recursos para la formación académica de los estudiantes (P.4.3; Sig. asintótica=0,418), las TD permiten una mejor elección en la selección de contenidos que usan los estudiantes (P.4.4; Sig. asintótica $=0,245)$, la comunicación e interacción entre los actores educativos a través de las TD hacen un aporte significativo al proceso educativo (P.5.6; Sig. asintótica=0,589), y la percepción que tienen de la Institución educativa al fomentar el uso de las TD (P.4.7; Sig. asintótica=0,640), como puede observarse en el Gráfico 11.

La adopción y uso de las tecnologías digitales en el profesorado universitario: un análisis desde la perspectiva del género y la edad. Byron Hidalgo-Cajo y Mercé Gisbert-Cervera. Página 12 de 19 


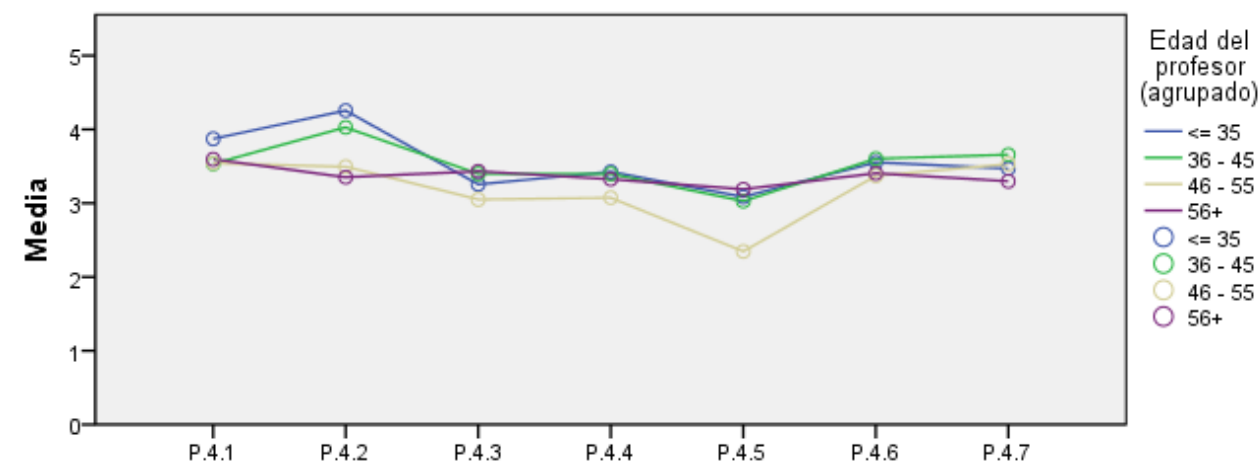

Gráfico 11. Percepción de las TD como herramienta didáctica (PU) en función de la edad

En cuanto a la Dimensión cinco. "Actitud hacia el uso de las aulas virtuales", medida en función de la Utilidad y Facilidad de uso de las AV, se puede destacar que en los intervalos de edad ( $<=35$ años), seguido de la edad mayores a 56 años ambas tienden a percibir mayor utilidad y facilidad hacia el uso de las AV en el proceso (E-A). Sin embargo, los intervalos de edad de (36-45 años) y (46-55 años) perciben un menor grado de la utilidad y facilidad de uso, Desde esta perspectiva se concluye, que no existen diferencias significativas en todos los ítems de esta dimensión, ver gráfico 12.

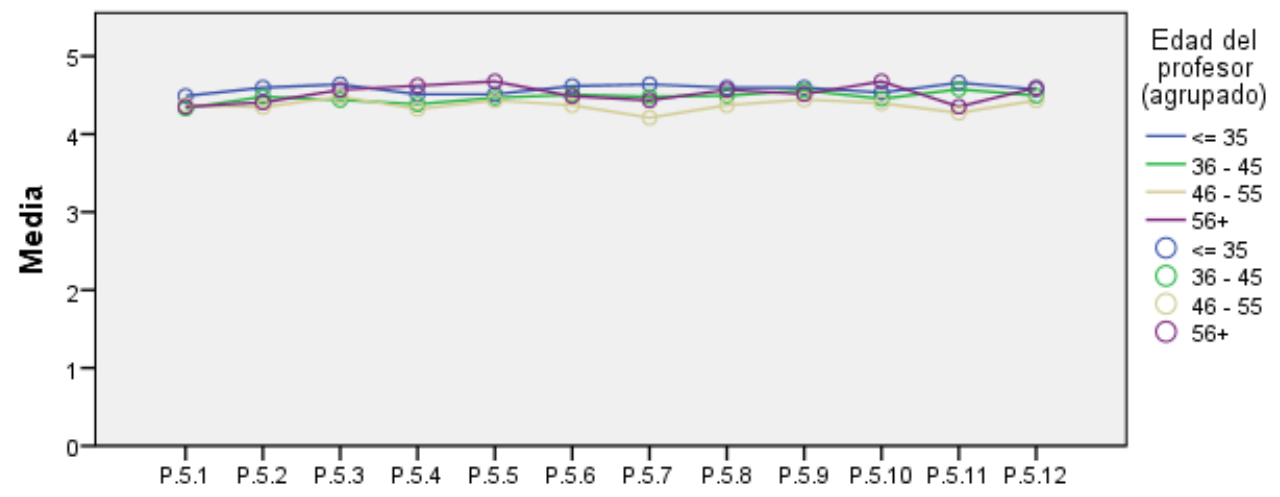

Gráfico 12. Actitud hacia el uso de AV en el proceso (E-A) en función de la edad

\section{Conclusiones y discusión de resultados}

El estudio realizado permite identificar el nivel de adopción, uso e integración de las TD autoevaluado por el profesorado universitario a partir de los datos recogidos con el cuestionario TAUT, los cuales se ajustan en las dimensiones de Propiedad y uso, Interés, Importancia, Percepción y actitud hacia el uso de las TD en el proceso (E-A) desde la perspectiva de las variables género y edad.

Al tener en cuenta las dimensiones del objeto de estudio, se puede mencionar de manera descriptiva que el profesorado tiene una actitud positiva respecto a la adopción y al uso de las TD en el proceso (E-A). Es así que el profesorado que ha participado en el estudio podemos caracterizarlo como un usuario "multidispositivo", ya que en su mayoría poseen, computadoras portátiles, de escritorio, tablet, lector de libros digitales, y smartphones, siendo las mujeres las que más dispositivos disponen y las que más los usan para propósitos educativos, así como para otras actividades.

La adopción y uso de las tecnologías digitales en el profesorado universitario: un análisis desde la perspectiva del género y la edad. Byron Hidalgo-Cajo y Mercé Gisbert-Cervera. Página 13 de 19 
Estos resultados son similares en las dimensiones; interés de uso de las TD, importancia que brinda el profesorado a las TD tanto dentro como fuera del aula de clase. Sin embargo en lo referente a la percepción que tiene el profesorado de las TD como herramienta didáctica y a la actitud respecto al uso de las $\mathrm{AV}$, los hombres son lo que más se destacan que las mujeres, resultados que son similares al estudio realizado por Cabezas et al. (2017).

Con el propósito de dar respuesta a las hipótesis planteadas, el estudio indagó sobre la influencia de las variables género y edad en relación a la adopción y uso de las TD en el profesorado universitario mediados por el cuestionario TAUT, en primera instancia se inicia con la variable género. ver Tabla 1.

Tabla 1.

Resumen Prueba U Mann-Witney en función del género

\begin{tabular}{|c|c|c|c|c|c|c|c|c|c|c|}
\hline & & & & & & & & GE & RO & \\
\hline & $\begin{array}{r}\text { Dim } \\
\text { Pr }\end{array}$ & $\begin{array}{l}\text { nsiones } \\
\text { leba t }\end{array}$ & $\begin{array}{r}\text { Lev } \\
\text { igua } \\
\text { var }\end{array}$ & $\begin{array}{l}\text { de } \\
\text { d de } \\
\text { zas }\end{array}$ & $\underset{\mathbf{m}}{\text { igua }}$ & $\begin{array}{l}\text { dad de } \\
\text { dias }\end{array}$ & HOI & BRE & & \\
\hline & & & $\mathbf{F}$ & Sig & $\mathbf{t}$ & \begin{tabular}{|c|} 
Sig \\
(bilateral)
\end{tabular} & $\mathbf{X}$ & SD & $\mathbf{X}$ & SD \\
\hline 1 & Propiedad & $\begin{array}{l}\text { Se asume varianzas iguales. } \\
\text { No se asume varianzas iguales. }\end{array}$ & 0,91 & 0,40 & $\begin{array}{l}0,44 \\
0,44\end{array}$ & $\begin{array}{l}0,67 \\
0,67\end{array}$ & 3,67 & 1,39 & 3,71 & 1,42 \\
\hline & Uso & $\begin{array}{l}\text { Se asume varianzas iguales. } \\
\text { No se asume varianzas iguales. }\end{array}$ & 0,85 & 0,47 & $\begin{array}{l}0,77 \\
0,76\end{array}$ & $\begin{array}{l}0,46 \\
0.46\end{array}$ & 2.70 & 0,93 & 2,75 & 0,95 \\
\hline 2 & Interés & $\begin{array}{l}\text { Se asume varianzas iguales. } \\
\text { No se asume varianzas iguales. }\end{array}$ & 0,95 & 0,45 & $\begin{array}{l}1,21 \\
1,22\end{array}$ & $\begin{array}{l}0,35 \\
0,35\end{array}$ & 3,51 & 1,11 & 3,68 & 1,06 \\
\hline 3 & Importancia TD (E-A) & $\begin{array}{l}\text { Se asume varianzas iguales. } \\
\text { No se asume varianzas iguales. }\end{array}$ & 1,09 & 0,35 & $\begin{array}{l}1,33 \\
1,34\end{array}$ & $\begin{array}{l}0,31 \\
0,31\end{array}$ & 2,94 & 1,31 & 3,18 & 1,26 \\
\hline & Importancia TD Aula & $\begin{array}{l}\text { Se asume varianzas iguales. } \\
\text { No se asume varianzas iguales }\end{array}$ & 0,99 & 0,51 & $\begin{array}{l}0,58 \\
0,45\end{array}$ & $\begin{array}{l}0,66 \\
0,67\end{array}$ & 2,79 & 1,26 & 2,80 & 1,34 \\
\hline 4 & Percepción de las TD & $\begin{array}{l}\text { Se asume varianzas iguales. } \\
\text { No se asume varianzas iguales. }\end{array}$ & 1,68 & 0.46 & $\begin{array}{l}0,80 \\
0,79\end{array}$ & $\begin{array}{l}0,47 \\
0,47\end{array}$ & 3,43 & 1,29 & 3,34 & 1,34 \\
\hline 5 & Actitud (Utilidad TD) & $\begin{array}{l}\text { Se asume varianzas iguales. } \\
\text { No se asume varianzas iguales. }\end{array}$ & 2,54 & 0,24 & $\begin{array}{l}1,55 \\
1,47\end{array}$ & $\begin{array}{l}0,25 \\
0,26\end{array}$ & 4,52 & 0,69 & 4.35 & 0,89 \\
\hline & Actitud (Facilidad TD) & $\begin{array}{l}\text { Se asume varianzas iguales. } \\
\text { No se asume varianzas iguales. }\end{array}$ & 2,30 & 0,17 & $\begin{array}{l}1,27 \\
1,23\end{array}$ & $\begin{array}{l}0,22 \\
0,23\end{array}$ & 4,53 & 0,70 & 4,39 & 0,83 \\
\hline
\end{tabular}

La influencia de la variable género en la adopción, uso e integración de las TD en el proceso (E-A) por parte del profesorado universitario ha sido objeto de estudio en distintas investigaciones. Unas señalan, la existencia de diferencias significativas según (Cabero et al., 2008; Liu y Sun, 2012; Barrantes Casquero et al., 2014), mientras que en otras no, (Hidalgo y Gisbert, 2021; Mercader, 2019; Muhaimin et al., 2020; Tafazoli et al., 2019; Ramírez et al., 2010; Hernández y Torrijos, 2019; Campos y Ramírez, 2018). De la misma manera, en la literatura científica se da a conocer que los hombres son los que mejor autoeficacia tienen en adoptar, usar e integrar las TD en el proceso (E-A); por lo contrario, otros estudios indican diferencias a favor de la mujer (Cózar et al., 2015). Como vemos en la literatura no existen evidencias concluyentes sobre la incidencia de las diferencias según en género. En nuestro caso, se determina que no existe diferencias significativas en función de la variable género con lo que respecta a la adopción, uso e integración de la TD en el proceso (E-A).

Del mismo modo, ha sido objeto de estudio la variable edad, que según Almerich et al. (2003) en la medida en la que se incrementa la edad del profesor disminuye el nivel de adopción de la tecnología (Martin et al., 2014; Martos et al., 2016).

La adopción y uso de las tecnologías digitales en el profesorado universitario: un análisis desde la perspectiva del género y la edad. Byron Hidalgo-Cajo y Mercé Gisbert-Cervera. Página 14 de 19 
Así también Vera et al. ( 2014) aseguran que la edad del profesorado modifica el nivel de uso de las TD, mientras que Cortés (2017) argumenta que el profesorado de mayor edad tiende hacer un menor uso de las TD y los de menor edad hacen mayor uso. Por lo contrario Mercader (2019) menciona que no se muestran diferencias significativas en función de la variable edad.

La Tabla 2 presenta el resumen del análisis estadístico en función de la variable edad en relación con la adopción, uso e integración de las TD en el proceso (E-A) por parte del profesorado, analizando de manera resumida las cinco dimensiones de estudio.

Tabla 2.

Resumen Prueba Kruskal - Wallis en función de la edad

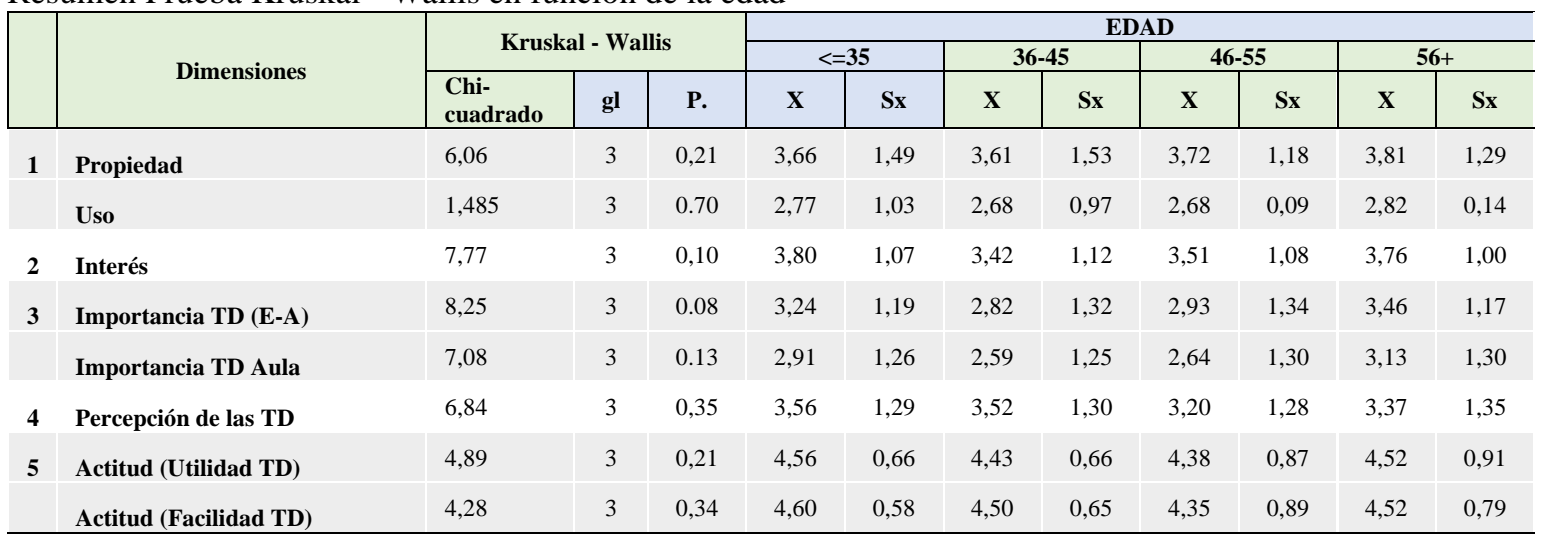

El estudio no ha encontrado diferencias significativas en función de la variable edad en relación con la adopción y uso de las TD en el proceso (E-A) por parte del profesorado universitario, resultados que son similares al estudio realizado por Campos y Ramírez (2018), quienes resaltan que la edad no influye en la adopción y uso de las TD, mientras que otros toman a la variable edad como un factor que influye en las intenciones del comportamiento hacia la adopción de las tecnologías (Leem y Sung, 2019).

En conclusión, las variables moderadoras género y edad que son analizadas en este estudio, se determina que son variables que no influyen en el proceso de adopción, uso e integración de las TD.

Presentación del artículo: 07 de mayo 2021 Fecha de aprobación: 14 de septiembre de 2021 Fecha de publicación: 30 de septiembre de 2021

Hidalgo, B. \& Gisbert, M. (2021) Adopción y uso de las tecnologías digitales en el profesorado universitario: un análisis de género y edad. RED. Revista de educación a distancia, 21(67). http://dx.doi.org/10.6018/red.481161

La adopción y uso de las tecnologías digitales en el profesorado universitario: un análisis desde la perspectiva del género y la edad. Byron Hidalgo-Cajo y Mercé Gisbert-Cervera. Página 15 de 19 


\section{Financiación}

Este trabajo no ha recibido ninguna subvención específica de los organismos de financiación en los sectores públicos, comerciales o sin fines de lucro

\section{Referencias bibliográficas}

Almerich, G., Suárez, J. M., Orellana, N., Gargallo, B., Belloch, C., Bo, R., Sáez, A., Aliaga, F., Gastaldo, I., \& Díaz, I. (2003). Teachers competencies in primary and secondary education. A, Méndez-Vilas, JA Mesa y J. Mesa (Eds.): Advances in Technology-Based Education: Towards and Knowledge Based Society. ÍCTE 2003, 2, 1045-1049.

Andoh Charles, B. (2012). Factors influencing teachers' adoption and integration of information and communication technology into teaching: A review of the literature. International Journal of Education and Development Using Information and Communication Technology, 8(1), 136-155. https://www.learntechlib.org/p/188018/

Araiza, M., \& Pedraza, E. (2019). Discernimiento de los docentes por género en el uso de las TIC en el aula a partir de las competencias digitales. Espacios, 40(21), 21-28.

Ariza, J. M. R., Barajas, E. R. G., \& Polanco, M. P. R. (2018). Assessment of ICT skills of university lecturers: A case in Chile. Pixel-Bit, Revista de Medios y Educacion, 52, 55-65. https://doi.org/10.12795/pixelbit.2018.i52.04

Barrantes Casquero, G., Casas García, L., \& Luengo González, R. (2014). Competencias Tecnológicas de los profesores de Infantil y Primaria de Extremadura en función del género. IE Comunicaciones: Revista Iberoamericana de Informática Educativa, 19, 3.

Cabero, J., Llorente, M. C., \& Puentes, A. (2008). Alfabetización digital: un estudio en la Pontificia Universidad Católica Madre y Maestra. Sevilla: Fortic.

Cabezas González, M., Casillas Mártin, S., Sanches Ferreira, M., \& Teixeira Diogo, F. L. (2017). ¿Condicionan el género y la edad el nivel de competencia digital? Un estudio con estudiantes universitarios. Fonseca, Journal of Communication, 15(15), 109. https://doi.org/10.14201/fjc201715109125

Campos Cruz, H., \& Ramírez Sánchez, M. Y. (2018). ICT in the educational processes of a Public Research Center. Apertura, 10(1), 56-70. https://doi.org/10.32870/ap.v10n1.1160

Cortés, C. T. (2017). El uso de las TIC en las prácticas académicas de los profesores de la Benemérita Universidad Autónoma de Puebla Use of ICT in Teachers' Academic Practices in the Benemérita Universidad Autónoma de Puebla. Redie., 19(3), 115-125. https://www.redalyc.org/pdf/155/15553204010.pdf

Cózar, R., del Valle, M., Hernández, J. A., \& Hernández, J. R. (2015). Uso de la realidad aumentada en la formacion inicial de maestros. Digital Education, O(27), 138-153. http://revistes.ub.edu/index.php/der/article/view/11622/pdf

Espinosa, H. R., Betancur, L. F. R., \& Aranzazu, D. (2014). Alfabetización informática y uso de sistemas de gestión del aprendizaje (LMS) en la docencia universitaria. Revista

La adopción y uso de las tecnologías digitales en el profesorado universitario: un análisis desde la perspectiva del género y la edad. Byron Hidalgo-Cajo y Mercé Gisbert-Cervera. Página 16 de 19 
de La Educacion $\quad$ Superior, $\quad 43(171), \quad$ 139-159. https://doi.org/10.1016/j.resu.2015.03.004

Flores, M., Ortega, M., \& Sánchez, M. (2021). Las nuevas tecnologías como estrategias innovadoras de enseñanza-aprendizaje en la era digital. Revista Electrónica Interuniversitaria de Formación Del Profesorado, 24(1), 29-42. http://revistas.um.es/reifop

Hernández Ramos, J. P., \& Torrijos Fincias, P. (2019). Percepción del profesorado universitario sobre la integración de las Tecnologías de la Información y la Comunicación (TIC) en las modalidades docentes. Influencia del género y la edad. Edmetic, 8(1), 0-2. https://doi.org/10.21071/edmetic.v8i1.10537

Hernández Sampieri, R. (2014). Metodologia de la Investigación (Sexta).

Hidalgo-Cajo, B. G., \& Gisbert-Cervera, M. (2021). Diseño y validación de un instrumento para evaluar la adopción de la tecnología digital en el profesorado universitario. Campus Virtuales , Revista Cientifica de Tecnología Educatíva, 10, 5167. http://www.uajournals.com/campusvirtuales/journal/19/3.pdf

Hidalgo Cajo, B. G., Hidalgo Cajo, D. P., \& Hidalgo Cajo, I. M. (2017). El impacto de las redes sociales como herramientas de comunicación, interacción y colaboración en el proceso enseñanza aprendizaje en la educación superior. SATHIRI, 12(104-113). http://revistasdigitales.upec.edu.ec/index.php/sathiri/article/view/56/96

INTEF. (2017). Marco común de competencia digital docente. Instituto Nacional de Tecnologías Educativas y Formación del Profesorado Madrid.

Isman, P. D. A. (2014). Turkish Online Journal of TURKISH ONLINE. Online Journal, $13(2)$.

Kafyulilo, A., Fisser, P., \& Voogt, J. (2016). Factors affecting teachers' continuation of technology use in teaching. Education and Information Technologies, 21(6), 15351554. https://doi.org/10.1007/s10639-015-9398-0

Leem, J., \& Sung, E. (2019). Teachers' beliefs and technology acceptance concerning smart mobile devices for SMART education in South Korea. British Journal of Educational Technology, 50(2), 601-613. https://doi.org/10.1111/bjet.12612

Liu, T., \& Sun, H. (2012). Gender Differences on Information Literacy of Science and Engineering Undergraduates. International Journal of Modern Education and Computer Science, 4(2), 23-30. https://doi.org/10.5815/ijmecs.2012.02.04

Lohbeck, A., Hagenauer, G., \& Frenzel, A. C. (2018). Teachers' self-concepts and emotions: Conceptualization and relations. Teaching and Teacher Education, 70, 111120. https://doi.org/10.1016/j.tate.2017.11.001

Luzardo Briceño, M., Sandia Saldivia, B. E., \& Soraya, A. J. A. (2020). Conocimiento y frecuencia del uso de las tecnologías de información y comunicación en la práctica educativa. Variables sociodemográficas de los docentes en la Universidad de Los Andes. http://scielo.sld.cu/pdf/rces/v39n1/0257-4314-rces-39-01-e3.pdf

Martin-Garcia, A. V, Hernandez-Serrano, J., \& Sánchez Gómez, M. C. (2014). Fases y clasificación de adoptantes de blended learning En Contextos Universitarios . 4, 457-

La adopción y uso de las tecnologías digitales en el profesorado universitario: un análisis desde la perspectiva del género y la edad. Byron Hidalgo-Cajo y Mercé Gisbert-Cervera. Página 17 de 19 
477.

Martos Sánchez, E., Pérez-García, P., \& Bernal-Vázquez, J. (2016). Relación entre la edad del profesorado de música andaluz y el desarrollo de la Escuela TIC 2.0. Revista $\begin{array}{llll}\text { Complutense } & \text { Educacion, } & \text { 27(2), }\end{array}$ https://doi.org/10.5209/rev_RCED.2016.v27.n2.48264

Matas-Terrón, A. (2016). Envejecimiento Activo Active Ageing Training " Needs for Spanish Population. Píxel-Bit. Revista de Medios y Educación., 48, 225-240.

McMillan, J., \& Schumacher, S. (2005). Investigación Educativa. Una ntroduccion conceptual. 128-129.

Mercader, C. (2019). Las resistencias del profesorado universitario a la utilización de las $\begin{array}{llll}\text { tecnologías Aligitales. } & \text { Aula }\end{array}$ https://doi.org/10.17811/rifie.48.2.2019.167-174

Muhaimin, A., Habibi, A., Mukminin, A., \& Hadisaputra, P. (2020). Science teachers' integration of digital resources in education: A survey in rural areas of one Indonesian province. Heliyon, 6(8), e04631. https://doi.org/10.1016/j.heliyon.2020.e04631

Nysveen, H., \& Pedersen, P. E. (2005). Explaining intention to use mobile chat services: moderating effects of gender. Journal of Consumer Marketing, 22(5), 247-256. https://doi.org/10.1108/07363760510611671

Pérez, Ó. M. M. G., Almenara, J. C., \& Sampedro, B. S. (2016). Valoraciones de la "Aceptación de la Tecnología de Formación Virtual" por profesores universitarios asistentes a un curso de formación virtual. Edutec. Revista Electrónica de Tecnología Educativa, 0(56).

Ramírez-Correa, P., Rondán-Cataluña, F. J., \& Arenas-Gaitán, J. (2010). Influencia del género en la percepción y adopción de e-learning: Estudio exploratorio en una universidad chilena. Journal of Technology Management and Innovation, 5(3), 129141. https://doi.org/10.4067/S0718-27242010000300010

Tafazoli, D., Parra, M. E. G., \& Abril, C. A. H. (2019). Attitude towards computerassisted language learning: Do gender, age and educational level matter? Teaching English with Technology, 19(3), 22-39.

Tarhini, A., Hone, K., \& Liu, X. (2014). Measuring the moderating effect of gender and age on E-learning acceptance in England: A structural equation modeling approach for an extended Technology Acceptance Model. Journal of Educational Computing Research, 51(2), 163-184. https://doi.org/10.2190/EC.51.2.b

Teo, T. (2012). Examining the intention to use technology among pre-service teachers: An integration of the Technology Acceptance Model and Theory of Planned Behavior. Interactive Learning Environments, 20(1), 3-18. https://doi.org/10.1080/10494821003714632

Vázquez-Cano, E., Meneses, E. L., \& García-Garzón, E. (2017). Differences in basic digital competences between male and female university students of Social Sciences in Spain. International Journal of Educational Technology in Higher Education, 14(1). https://doi.org/10.1186/s41239-017-0065-y

La adopción y uso de las tecnologías digitales en el profesorado universitario: un análisis desde la perspectiva del género y la edad. Byron Hidalgo-Cajo y Mercé Gisbert-Cervera. Página 18 de 19 
Vera, J., Torres, L., \& Martínez, E. (2014). Evaluación de competencias básicas en TIC en docentes de educación superior en México. Pixel-Bit. Revista de Medios y Educación, 44, 143-155. http://www.redalyc.org/articulo.oa?id=36829340010

Wong, K. T., Teo, T., \& Russo, S. (2012). Influence of gender and computer teaching efficacy on computer acceptance among Malaysian student teachers: An extended technology acceptance model. Australasian Journal of Educational Technology, 28(7), 1190-1207. https://ajet.org.au/index.php/AJET/article/view/796/96 